\title{
Coherence-imaging approach to time-resolved charge-exchange recombination spectroscopy in high-temperature plasma
}

\author{
J. Howard ${ }^{\text {a) }}$ \\ Plasma Research Laboratory, Australian National University, Canberra ACT 0200, Australia \\ L. Carraro, M. E. Puiatti, F. Sattin, P. Scarin, M. Valisa, and B. Zaniol \\ Consorzio RFX, Associazione Euratom-Enea sulla Fusione, Corso Stati Uniti 4-35127, Padova, Italy \\ R. König and J. Chung \\ Max-Planck-Institut für Plasmaphysik, Teilinstitut, Greifswald, Germany
}

(Presented on 10 July 2002)

\begin{abstract}
A coherence-based, or interferometric approach to spectral analysis of charge-exchange recombination (CXR) emission radiation from high-temperature plasma probed or heated using energetic neutral beams, offers a number of advantages over wavelength-domain instruments. The spectral-line shift and broadening are obtained from measurements of the spectral coherence at a given fixed time delay. The coherence is monitored by first approximately isolating the spectral line of interest using an interference filter and subsequently imaging the spectral scene using a field-widened electro-optic path-delay-modulated polarization interferometer. Interferometers have the advantage of high-light throughput (no slit aperture). Moreover, because the spectral information is encoded at harmonics of the electro-optic modulation frequency, a single detector suffices to capture the spectral information, thereby opening the possibility for time-resolved two-dimensional spectral imaging. When unwanted spectral features are passed by the interference filter, the interpretation of the coherence phase and amplitude images can become ambiguous. By modulating the particle beam source, however, we show that coherence imaging using a single-delay modulatable interferometer can distinguish and characterize the Doppler-broadened CXR emission component against a significant background of continuum and intrinsic radiation, or pollution from nearby spectral features. (C) 2003 American Institute of Physics. [DOI: 10.1063/1.1537036]
\end{abstract}

\section{INTRODUCTION}

Charge-exchange recombination spectroscopy (CXRS) is a powerful tool for obtaining spatially resolved plasma temperature and flow measurements in high-temperature plasma magnetic confinement devices. ${ }^{1,2}$ The method is based on Doppler broadening of emission line radiation from plasma impurities which radiate after obtaining an electron from diagnostic or heating neutral particle beams injected into the plasma. Nevertheless, time resolution can be limited both by charge coupled device $(\mathrm{CCD})$ readout rate and the loss of light necessitated by the grating spectrometer input slit. More importantly, only one spatial dimension can be imaged because one axis of the CCD array is required to capture the spectrally dispersed light. It is usual to use a fitting procedure to match a Doppler-broadened and shifted Gaussian line shape to the measured spectrum to extract lineof-sight averaged temperatures and projected flow speeds. More sophisticated methods, such as modulating the beam source can be useful when additional contributions from the cooler plasma edge or other spectral contaminants are important.

The optical throughput advantages of time-domain interferometers for spectroscopy are well known. ${ }^{3}$ However, the necessity to scan the optical path length to obtain the Fourier

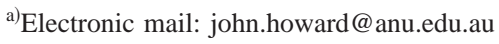

transform, and the stringent mechanical constraints for operation at optical wavelengths have negated this advantage. When the spectral information content is small, however, a modulated fixed delay interferometer can encode the unknown spectral parameters at harmonics of the modulation frequency. Based on this idea, high-throughput, twodimensional (2D) time-resolved "coherence-imaging" systems have recently been reported for Doppler spectroscopy of intrinsic plasma emission. ${ }^{4}$ The fixed optical delay and path length modulation can be obtained using solid electrooptic and birefringent plates configured as a polarization interferometer (the so-called MOSS spectrometer). It is important to note that the utility of the device relies on the availability of a realistic model of the received spectrum that requires only a small number of free parameters for its description. In the absence of a model, or for more complex or ambiguous spectra, well-established grating-based methods remain essential.

In this article, we consider the applicability of 2D coherence-imaging systems for high-temperature plasma spectroscopy. In many scenarios, spectral contamination either by continuum radiation or other spectral lines is unavoidable. Spectral line asymmetries arising, for example, from energy-dependent charge-exchange cross sections, can also complicate interpretation. In such cases, multiple crystal wave plates can be used to monitor the coherence simultaneously at a number of delays. Given an appropriate model 


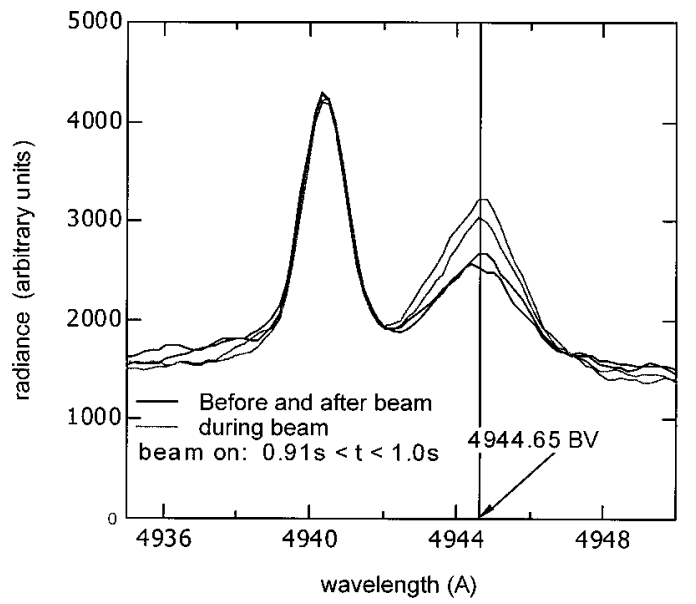

FIG. 1. Experimentally measured CX spectrum showing enhancement of the BV emission during the neutral beam injection. (Courtesy R. Granetz).

for the spectrum, the multiple-delay measurements allow extraction of the unknown model parameters. ${ }^{4}$ For modulated diagnostic neutral beam CXR studies, we show here, however, that it is possible to extract the Doppler broadened and shifted CX component from an arbitrary background using coherence measurements at just a single optical delay.

We also report some recent instrument design and concept advances presently being incorporated into MOSS camera systems for modulated CXRS measurements on the Alcator C-MOD tokamak and for divertor studies in the Wendelstein 7-AS stellarator. The systems feature wide-field temperature-compensated modulator plates and thermally compensated compound fixed-delay wave plates. By mating MOSS with a suitably synchronized fast framing camera, two-dimensional, time-resolved Doppler spectral imaging of plasma light is feasible. This could be a particularly important diagnostic capability for the next generation of toroidally asymmetric devices.

\section{COHERENCE-IMAGING FOR MODULATED CX DOPPLER SPECTROSCOPY}

In the case of a spectrally isolated Doppler broadened line, the interferogram contrast (or fringe visibility) gives a line integral of the emission weighted temperature distribution (approximately independent of the flow), while the interferogram phase yields a line integral of the weighted plasma flow component in the direction of view. ${ }^{5}$ However, it is not always the case that a simple spectral line can be isolated from contaminating components and background. When continuum emission or other features pollute the spectrum, special measures must be adopted to relate the measured fringe coherence to unknown source parameters. For example, if the light-emission source can be modulated using a diagnostic neutral beam, the "beam-on" and "beam-off" components can be very simply separated, without the need for spectral line fitting procedures.

We illustrate the CXRS problem with reference to spectral data obtained on the Alcator C-MOD tokamak using a diagnostic neutral beam to study the $494.5 \mathrm{~nm}$ emission line from hydrogenlike boron. ${ }^{6}$ The spectrum, which is shown in Fig. 1, is comprised of continuum, a BII contamination line and $\mathrm{BV}$ emission in both the presence and absence of the diagnostic beam.

The interferogram for each of these terms is of the form

$$
S_{i}=\frac{I_{i}}{2}\left[1+\zeta_{i} \cos \left(\phi_{i}+\tilde{\phi}\right)\right],
$$

where $\tilde{\phi}$ is the time varying electro-optic modulation. With some simple algebra, the total interferogram can be expressed in the same form

$$
S_{p}=\sum_{i} S_{i}=I_{p}\left[1+\zeta_{p} \cos \left(\phi_{p}+\tilde{\phi}\right)\right]
$$

where $I_{p}, \zeta_{p}$, and $\phi_{p}$ are the effective intensity, contrast, and phase from the plasma emission alone (beam off). These composite quantities depend on the details of the transmitted spectrum but can nevertheless be measured unambiguously by the MOSS camera during the beam-off phase. We characterize the plasma background spectral information by constructing from the experimentally determined parameters the "complex coherence" $\gamma_{p}=I_{p} \zeta_{p} \exp \left(I \delta_{p}\right)$ where $\delta_{p}=\phi_{p}$ $-\phi_{0}$ is the interferogram phase shift referred to a dc phase bias which, for convenience, we take as the birefringent phase delay $\phi_{0}=2 \pi L B\left(\nu_{0}\right) \nu_{0} / c$ where $L$ is the delay plate thickness and $B\left(\nu_{0}\right)$ is the birefringence at the spectral line center frequency $\nu_{0}$.

When the beam is on, we obtain the total interferogram

$$
S_{b}=S_{p}+S_{\mathrm{cx}}
$$

where $S_{\mathrm{cx}}$ is also of the form Eq. (1) and represents the charge-exchange component. Once again, we construct the complex coherence for the beam-on phase, $\gamma_{b}$ $=I_{b} \zeta_{b} \exp \left(I \delta_{b}\right)$, and write for the coherence of the chargeexchange component $\gamma_{\mathrm{cx}} \equiv\left(I_{b}-I_{p}\right) \zeta_{\mathrm{cx}} \exp \left(I \delta_{\mathrm{cx}}\right)$. The fringe contrast and the interferometric phase for the chargeexchange emission are now trivially obtained from the absolute value and phase of the complex quantity

$$
\zeta_{\mathrm{cx}} \exp \left(I \phi_{\mathrm{cx}}\right)=\frac{\gamma_{b}-\gamma_{p}}{I_{b}-I_{p}} .
$$

Ignoring line-integration effects, and after accounting for the instrument contrast, the fringe visibility gives the temperature $T_{\mathrm{cx}}$ of the charge-exchange ions and the phase shift gives the projected flow speed $v_{\mathrm{cx}}=\boldsymbol{v}_{\mathrm{cx}} \cdot \hat{\boldsymbol{l}}$ in the direction of view: ${ }^{5}$

$$
\begin{aligned}
& \zeta_{\mathrm{cx}}=\exp \left[-T_{\mathrm{cx}} / T_{C}\right] \\
& \delta_{\mathrm{cx}}=\hat{\phi}_{0} v_{\mathrm{cx}}
\end{aligned}
$$

where the "characteristic temperature" $T_{C}$ is given by

$$
k T_{C}=\frac{1}{2} m v_{C}^{2}
$$

with "characteristic velocity" set by the effective interferometer phase delay $\hat{\phi}=\kappa \phi_{0}$

$$
v_{C}=\frac{2 c}{\hat{\phi}_{0}} \text {. }
$$

Here, $m$ is the mass of the radiating ion and 


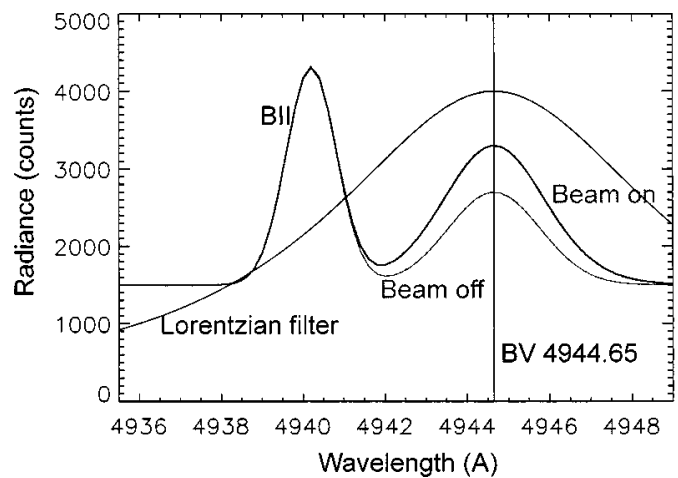

(a)

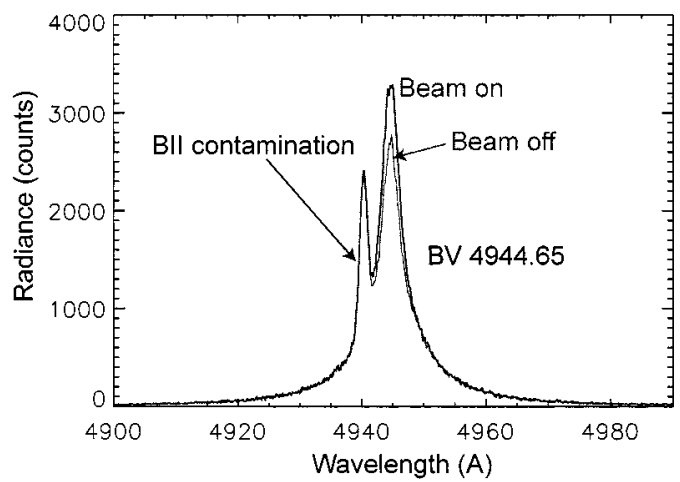

(b)

FIG. 2. (a) The model spectrum used for these simulations showing BII contamination and BV charge-exchange line. A representative Lorentzian bandpass filter of FHWM $1 \mathrm{~nm}$, and centered on the BV line, is shown for comparison. (b) The resulting filtered and noise-corrupted spectrum.

$$
\kappa=1+\frac{\nu_{0}}{B_{0}} \frac{\partial B}{\partial \nu}
$$

accounts for the optical frequency dispersion of the birefringence.

From Eq. (4), it can be seen that estimation of the charge-exchange coherence $\gamma_{\mathrm{cx}}$ will depend sensitively on the intensity difference $I_{b}-I_{p}$. To improve the reliability of the measurement it would seem important to reduce the continuum contribution by using an appropriate bandpass filter. However, the inferred temperature will also depend on the ratio of the Doppler linewidth to the filter passband. We have modeled the MOSS camera response to a BV spectrum constructed to closely match the features observed in Fig. 1, but subject to Lorentzian spectral filters of various width. We have performed a statistical analysis to compare the ability of dispersive and coherence-based systems to characterize the beam-generated light emission. For the purposes of the analysis, we ignore instrumental components, and assume both systems are equally light efficient. The beam-induced emission is modeled as a Doppler broadened contribution of amplitude 800 counts and temperatures between 600 and $1400 \mathrm{eV}$. Light signals are corrupted with shot noise appropriate to the photoelectron count and an ensemble of 50 realizations are averaged to obtain mean temperatures and their standard deviations. Doppler shifts have not been considered.

The model spectrum and representative filtered optical spectra are shown in Fig. 2. The associated fringe visibilities

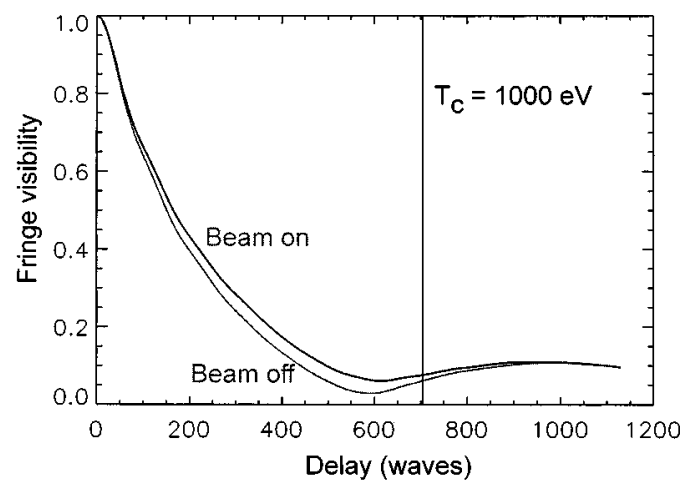

(a)

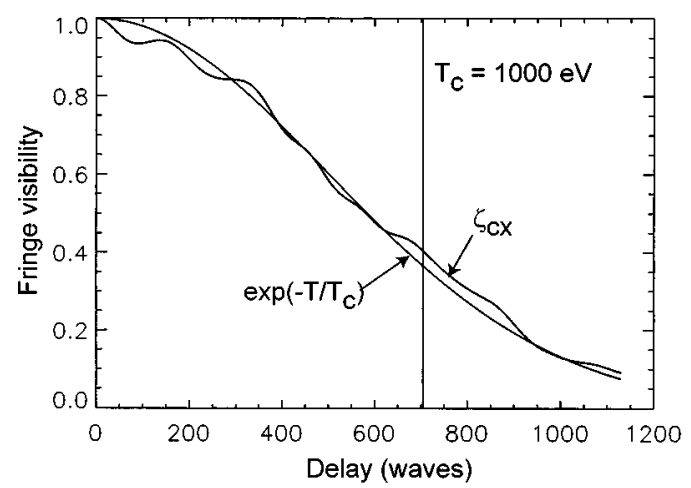

(b)

FIG. 3. (a) The fringe visibility for the filtered spectrum of Fig. 2(b) for beam-on and -off cases. The vertical line indicates the delay corresponding to $T_{c}=1000 \mathrm{eV}$. (b) The extracted fringe visibility for the charge-exchange component alone. The theoretical curve shows the contrast variation expected for a $1 \mathrm{keV}$ Doppler-broadened emission line.

(beam-off and -on states) calculated from the noise-corrupted spectra, and the charge-exchange related component inferred from Eq. (4) are shown in Fig. 3. The initial decrease of the visibility at delays less than 300 waves is dictated primarily by the width of the total transmitted spectrum, with the first minumum corresponding to a destructive interference of the $\mathrm{BII}$ and BV contributions. We assume a MOSS system having characteristic temperature $T_{c}=1000 \mathrm{eV}$ corresponding to a delay of approximately 700 waves (vertical line in Fig. 3) and calculate the fringe contrast $\zeta_{\mathrm{cx}}$ for temperatures in the range of 600 to $1400 \mathrm{eV}$ in $200 \mathrm{eV}$ steps. These simulations are repeated for interference filters having full width half maxima 1.0, 1.5, 2.5, and $5.0 \mathrm{~nm}$.

Figure 4 shows the inferred temperature normalized to true temperature plotted as a function of the $\mathrm{BV}$ spectral line full width at half maximum (FWHM) (or temperature) normalized to the applied filter FWHM. The error bars are two standard deviations in width. The solid curve shows the expected normalized inferred temperature variation as a function of Doppler to filter FWHM ratio in the absence of noise. As expected, the temperature estimate improves with the rejection of the contamination, but must be corrected for the bias introduced by filter apodization. We have not explored alternative filter strategies or operation at different optical delays.

For comparison, we have fitted a Gaussian profile to the difference between the beam-on and -off spectra. The results 


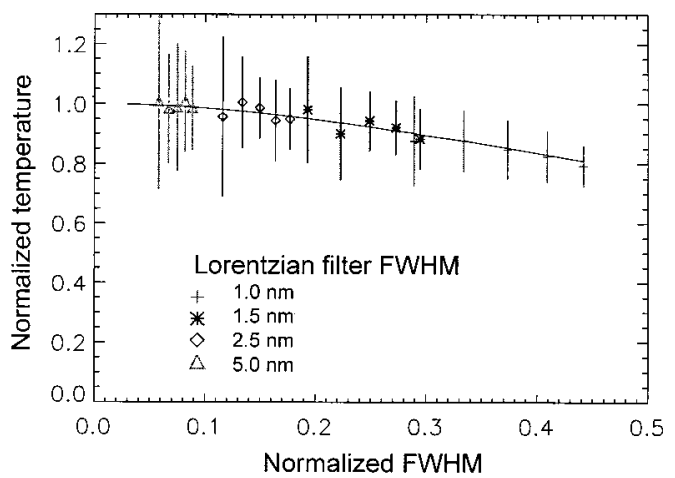

(a)

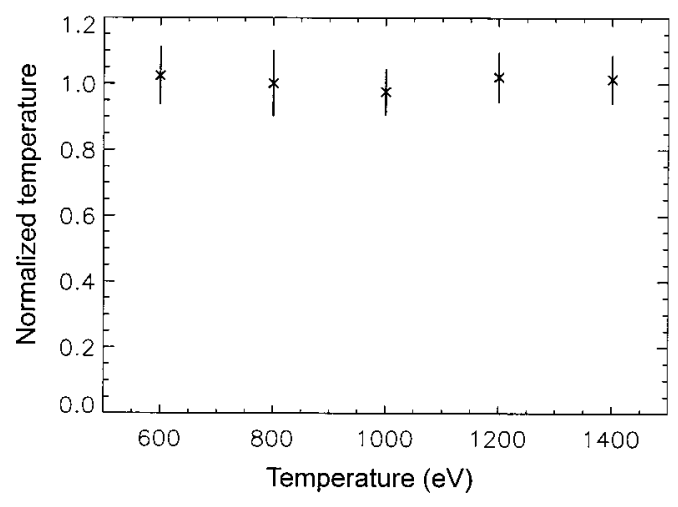

(b)

FIG. 4. Results of statistical analysis. (a) The ratio of inferred to true temperature vs normalized Doppler width for a number of bandpass filter widths and temperatures in the range of 600-1400 eV. Measurement reliability decreases for larger filter widths where the fractional modulation of the light intensity produced by the beam is small. (b) Normalized inferred temperature vs true temperature for wavelength domain instrument. See text for discussion.

shown in Fig. 4(b) show a comparable or slightly better noise performance for the dispersive instrument. However, it is likely this would be more than offset by the greater light efficiency for the 2D coherence imaging system. We conclude that a single delay (i.e., single birefringent plate) coherence imaging system is capable of distinguishing the beam component parameters, irrespective of the background contamination, in the case where the diagnostic beam can be modulated and that the plasma does not change significantly in one modulation period.

\section{INSTRUMENT DESIGN CONCEPTS AND RECENT DEVELOPMENTS}

It is a property of lithium niobate, the electro-optic, and birefringent material used as both modulator and delay plate in earlier versions of the MOSS camera, that the phase delay $\phi_{0}$ drifts slowly in time due to ambient temperature variations. While this is not an issue for modulated beam experiments, for spectroscopy on long plasma pulse devices it is important that the optical delay remain constant.

To overcome this problem, it is advantageous to separate the modulator and optical delay functions. Temperature- compensated lithium niobate modulators can be constructed by crossing two equithickness plates (to cancel the net birefringence) and reversing the polarities of the modulating voltage. ${ }^{7}$ Using this approach, for modulator plates of a thickness of $25 \mathrm{~mm}$, we have been able to reduce ambient temperature-related phase drifts from around one wave in an hour to less than $5 \mathrm{mrad}$. Unfortunately, the static residual birefringence for off-axis rays can result in a significant loss of fringe contrast for imaging systems. This loss can be largely eliminated by combining two such temperaturecompensated modulators with their optical axes orthogonal and with an intervening half wave plate having fast-axis oriented at $45^{\circ}$ to each.

Temperature-drift compensated compound delay plates will be constructed using birefringent crystals having different material properties.

For a prescribed delay offset $\phi_{0}$ the crystal thicknesses are determined by solving the trivial simultaneous equations

$$
\begin{aligned}
& \phi_{01}+\phi_{02}=\phi_{0}, \\
& \alpha_{1} \phi_{01}+\alpha_{2} \phi_{02}=0,
\end{aligned}
$$

where the birefringent phase delay introduced by the $i$ th plate is given by $\phi_{0 i}=2 \pi L_{i} B_{i} / \lambda_{0}$. A number of highbirefringence uniaxial birefringent materials, including lithium niobate, yttrium orthovanadate (YVO4), and $\alpha$-BBO are suitable for this purpose.

We have constructed a time-resolved coherence-imaging camera for study of $468 \mathrm{~nm}$ He II emission from the divertor regions in the W7-AS stellarator. Successive quadrature images of the interferogram are obtained by locking the electrooptic plate modulation with the frame rate of a commercially available fast-framing CCD camera (PCO Sensicam LE). Synchronization is achieved by using the camera exposure timing pulses to clock a programmable digital-to-analog converter output staircase wave which is amplified (1000x) before being applied to the modulator electrodes. The staircase, which is designed to produce phase steps $(0, \pi / 2, \pi, 3 \pi / 2)$, is generated using a custom LabVIEW-based application that both controls a National Instruments PCI-MIO-16-E4 PCcard and communicates with the CCD camera. The camera can be configured for simultaneous readout and exposure to obtain close to a $100 \%$ operational duty cycle. Following automatic compensation for the instrument response, sets of three or four sequential frames can be processed to deliver the interferogram intensity, contrast, and phase.

${ }^{1}$ R. C. Isler and L. E. Murray, Appl. Phys. Lett. 42, 355 (1983).

${ }^{2}$ R. J. Fonck, D. S. Darrow, and K. P. Jaehnig, Phys. Rev. A 29, 3288 (1984).

${ }^{3}$ P. Jacquinot, J. Opt. Soc. Am. 44, 761 (1954).

${ }^{4}$ J. Howard, C. Michael, F. Glass, and A. Cheetham, Rev. Sci. Instrum. 72, 888 (2001).

${ }^{5}$ J. Howard, Appl. Opt. 41, 197 (2002).

${ }^{6} \mathrm{R}$. Granetz (private communication).

${ }^{7}$ M. R. Biazzo, Appl. Opt. 10, 199 (2000). 